\title{
Research on Optimal Design of Driving Controller for Continuous Software Robot
}

\author{
Lin Rongxia, Lu Xinghua ${ }^{*}$, Fan Yuchang, Lin Xiaohong \\ Huali College Guangdong University of Technology, Guangdong Guangzhou, 511325, China \\ *Corresponding author E-mail: xhlu@gdut.edu.cn
}

Keywords: driving link; control; continuous flexible robot; optical sensing; distance measurement

\begin{abstract}
Aiming to improve the precision of driving link control of continuous software robot, a driving controller is designed, and a driving control law of continuous software robot based on dynamic inversion integral error compensation is proposed. Controller design mainly includes control algorithm design and controller hardware interface design. The control law of dynamic inversion integral error compensation is designed to improve the quality of driving link control of continuous soft robot. The central controller, driver module, optical ranging module and driving module of driving link controller are integrated. The optimization design of driving controller for continuous software robot is realized. The experimental results show that the controller has higher precision, better robustness and error correction ability, and improves the precision of motion control of the software robot, and the response time of the control process is shorter. The stability of the robot driving rod is guaranteed.
\end{abstract}

\section{Introduction}

With the development of intelligent control technology, the research of high-precision mechanical equipment and robots to replace manual work, improve the efficiency and accuracy of industrial operations and reduce the cost of labor has shown its unique advantages. Driving link, also known as manipulator, is an important part of continuous software robot ${ }^{[1]}$. Driving link can replace human to carry out high-precision mechanical work, such as UV irradiation, parts placement, screw locking, circuit board cutting and so on. When driving connecting rod is used in mechanical operation, it is necessary to accurately measure the working surface and the target position of the operation, and the driving link is usually used to measure the position of the operating position by optical sensor ranging method. Combined with intelligent control sensor of actuator, direct and control driving linkage operation. Therefore, it is of great significance to study the design method of driving controller for continuous software robot, which is of great significance in improving the precision and efficiency of driving link, and the related control algorithms and controller design have been paid great attention to.

The design of optical distance controller for driving link is of great significance in improving the stability of driving rod and improving the performance of driving link of continuous soft robot ${ }^{[2]}$. At 
present, the main control methods are fuzzy PID control method, synovium control method and inverse integral control method. The control scheme of fixed controller gain planning (Gain-Scheduling) is adopted. The parameters of the robot drive controller are corrected by distortion and error compensation, and the better control effect is obtained ${ }^{[3]}$. In reference [4], the driving link control method based on delay method and high precision pulse optical sensor distance measurement is designed, and the GPS information data of optical sensing ranging are written in the camera video based on the high-definition camera with automatic focus. The optical sensor ranging time of driving link and the extension angle of driving link can be controlled, which can effectively improve the error and control delay of optical sensor ranging, but the related hardware design technology is not mature. There are errors in the design of the distance measuring route of driving connecting rod, which need to be studied in depth ${ }^{[5]}$. To solve the above problems, this paper presents a driving control law for continuous software robot based on dynamic inversion integral error compensation.

\section{Driving control law of continuous software robot}

\subsection{Mathematical model of driving control for continuous software robot}

The construction of driving control system of continuous software robot is based on the mathematical model of driving linkage ${ }^{[6]}$. In order to deal with the problem conveniently, some simplified treatments are made in modeling: (1) the shape of driving rod is symmetrical about $x_{1} O y_{1}$ plane. (2) The damping force and position force of driving link are linearly changed. (3) The errors caused by the machining and installation of the soft robot motion control system are ignored. The attitude motion equation of soft robot motion control can be decomposed into two groups of dynamic motion equations of longitudinal motion and lateral motion, and the nonlinear equations of attitude dynamics of soft robot motion control driven by connecting rod are obtained as:

$$
\left\{\begin{array}{l}
m \dot{V}=-m g \sin \theta-c_{x} q S_{M}+P \\
m V \dot{\theta}=-m g \cos \theta+c_{y}^{\alpha} q S_{M} \alpha+P\left(\alpha+\delta_{\varphi}\right)+m_{R} l_{R} \ddot{\delta}_{\varphi} \\
J_{z 1} \ddot{\varphi}=-c_{y 1}^{\alpha} q S_{M}\left(x_{g}-x_{T}\right) \alpha-q S_{M} m_{d 2} l_{k}^{2} \dot{\varphi} / V-P\left(x_{R}-x_{T}\right) \delta_{\varphi}-m_{R} \dot{W}_{x 1} l_{R} \delta_{\varphi}-m_{R} l_{R} \ddot{\delta}_{\varphi}\left(x_{R}-x_{T}\right)-J_{R} \ddot{\delta}_{\varphi}
\end{array}\right.
$$

When the input variable is determined by driving the connecting rod, the state quantity of the stability characteristic functional of the output is obtained as $x_{0}\left(x_{0}=\left[\varphi_{0}, \dot{\varphi}_{0}, \theta_{0}\right]^{T}\right)$. According to the feedback correction of measurement error, the output balance condition of driving control value of continuous software robot is obtained. $f\left(x_{0}, u_{0}\right)=0$. Thus, the linearized small disturbance equation of the stability control of the driving link is obtained as follows:

$$
\left\{\begin{array}{l}
m V \Delta \dot{\theta}=\left(c_{y}^{\alpha} q S_{M}+P\right) \Delta \alpha+m g \sin \theta \Delta \theta+P \Delta \delta_{\varphi}+m_{R} l_{R} \Delta \ddot{\delta}_{\varphi}+F_{g r} \\
J_{z 1} \Delta \ddot{\varphi}=-c_{y 1}^{\alpha} q S_{M}\left(x_{g}-x_{T}\right) \Delta \alpha-q S_{m} m_{d z} l_{k}^{2} \Delta \dot{\varphi} / V-P\left(x_{R}-x_{T}\right) \Delta \delta_{\varphi}-m_{R} \dot{W}_{x 1} l_{R} \Delta \delta_{\varphi}-m_{R} l_{R} \Delta \ddot{\delta}_{\varphi}\left(x_{R}-x_{T}\right)-J_{R} \Delta \ddot{\delta}_{\varphi}+M_{g r}
\end{array}\right.
$$

In order to construct the motion control algorithm of software robot based on nonlinear strict feedback, according to the above mathematical model analysis of driving linkage ${ }^{[7]}$, the control number mathematical model of continuous software robot driving control is described as follows:

$$
\left\{\begin{array}{l}
\Delta \dot{\theta}=c_{1} \Delta \alpha+c_{2} \Delta \theta+c_{3} \Delta \delta_{\varphi}+c_{3}^{\prime \prime} \Delta \ddot{\delta}_{\varphi}+\bar{F}_{g r} \\
\Delta \ddot{\varphi}+b_{1} \Delta \dot{\varphi}+b_{2} \Delta \alpha+b_{3} \Delta \delta_{\varphi}+b_{3}^{\prime \prime} \Delta \ddot{\delta}_{\varphi}=\bar{M}_{g r} \\
\Delta \varphi=\Delta \theta+\Delta \alpha
\end{array}\right.
$$


The known coefficients of the model and the input of control parameters of the driving link in the radial channel of each operation are represented respectively.

\subsection{Design of control law for robot driving controller}

On the basis of the above mathematical model of driving link control, the quality of driving link control of continuous soft robot is improved by designing nonlinear strict feedback error correction control law. Let the learning step size be $\eta$. after step training and learning, the iterative equation of the weights of the driving link optical sensor ranging control neural network is obtained as follows:

$$
W(n+1)=W(n)-\eta \frac{\partial E}{\partial W}+\partial \Delta W(n)
$$

Based on the stability principle of PID control, the weights of the driving link in the iterative range of the learning step size of the optical sensor range compensation are obtained as follows:

$$
\frac{\partial E}{\partial w_{i j}}=\frac{\partial E}{\partial v} \frac{\partial v}{\partial x^{\prime \prime}} \frac{\partial x^{\prime \prime}}{\partial u^{\prime \prime}} \frac{\partial u^{\prime \prime}}{\partial I^{\prime \prime}} \frac{\partial I^{\prime \prime}}{\partial x_{j}^{\prime}} \frac{\partial x_{j}^{\prime}}{\partial u_{j}^{\prime}} \frac{\partial u^{\prime}}{\partial I_{j}^{\prime}} \frac{\partial I_{j}^{\prime}}{\partial w_{i j}}=-\frac{1}{m} \sum_{k=1}^{m} \delta^{\prime}(k) w_{j}^{\prime} \operatorname{sgn} \frac{u_{j}^{\prime}(k)-u_{j}^{\prime}(k-1)}{I_{j}^{\prime}(k)-I_{j}^{\prime}(k-1)} x_{i}(k)=-\frac{1}{m} \sum_{k=1}^{m} \delta_{j}(k) x_{i}(k)
$$

With nonlinear strict feedback, a time-delay closed-loop controller for static neuron control is constructed. The error adjustment iterative formula of motion control of soft robot is $r(k) \in R^{n}$, the input sequence of sensor information of optical sensor ranging $R^{n}$ is excited for a long time, as:

$$
w_{s j h}^{\prime}\left(n_{0}+1\right)=w^{\prime}\left(n_{0}\right)-\eta_{s j h}^{\prime} \frac{\partial J}{\partial w_{s j h}^{\prime}}
$$

With the design of the control law above, the error compensation of driving link control is realized, and the control algorithm is optimized. As the software embedding algorithm of the controller, the controller is designed through the program loading of the algorithm.

\section{Hardware design of driving controller for linkage robot}

\subsection{Overall design of controller}

On the basis of the improved design of the control algorithm, the algorithm is embedded in the DSP chip to design the hardware circuit of the controller for driving the connecting rod ${ }^{[8]}$. The modularization design of controller mainly includes central controller, driver module, optical ranging module and drive module. The driving link soft robot motion controller is composed of two parts: upper and lower machine position. The output transmission of the device in the controller is carried out by VIX bus technology, at the same time, the multi-channel signal is collected by optical sensor. The signal is processed and stored in real time and transmitted to the logic circuit module of the actuator in the output interface to guide the driving rod to operate. A direct control unit for driving a flexible robot, including a robot motion control collector, a direct controller, and a display screen. In the driver module of the controller, the controller chooses PCI9054 as the bridge chip of the PCI, uses the PCI bridge chip to realize the high-speed data communication between the PC computer and the DSP, and adopts 32-bit or 64-bit data lines, which can effectively use the bandwidth of the bus to transfer. The state and environment data of software robot motion control are collected, and the control scheme is given. The overall structure of the controller is shown in figure 1. 


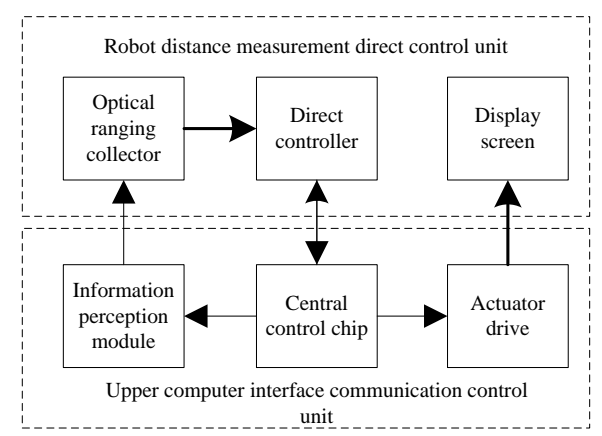

Fig. 1 Overall structure block diagram of controller

\subsection{Modular design of controller}

According to the overall design scheme, the modularization design of controller is carried out, and the modular design of motion controller of soft robot driven by connecting rod is carried out by using embedded design technology. The design criterion of the control law mentioned above is combined with the design specification. In the selection of peripheral devices, a 32-bit embedded control chip named GT8340 is selected as the peripheral device to carry out embedded design. GT8340 has the function of cache, the maximum working frequency is $1000 \mathrm{MHz}$. On the basis of the selection of peripheral devices, the main control chip is selected. The main control chip uses TMS320VC5509A DSP chip, which is a low power 16-bit fixed-point DSP, which is more suitable for the application of driving link control environment. On this basis, the modularization design of the controller is carried out. There are three working modes of the central controller, Con M and J, respectively. The system requires high precision of the conversion frequency for the motion control of the software robot, so it is in the central controller. Three working modes are used for logic conversion. ADM706SAR, is programmed by CPLD to communicate directly with other C54X devices in the system. The clock and frame synchronization signal is interrupted to CPU, to realize the program drive and control output of the control center through high-speed A/D. The interface design of the actuator adopts serial port communication scheme, configures the sampling rate generating register, reads the data from the serial port receiving data register, waits for two clock cycles, starts the internal frame synchronization signal, and in the interrupt service program, Handle control interrupt events. Based on the above description, the integrated circuit of the driving link robot driver controller designed in this paper is shown in figure 2.



Fig. 2 Hardware design of driving controller for driving linkage robot

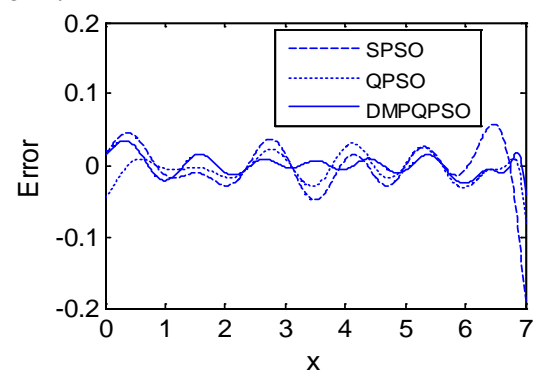

Fig. 3 Control performance comparison

\section{Experimental test analysis}

Aiming to analyze the performance of the soft robot motion controller designed in this paper, we need to carry out an experiment to analyze the actual control. In the experiment, different driving linkage control models are put in the same position. The experimental results were analyzed and 
discussed. The driving link used in the experiment is the driving link of Robot ARM used in a national key laboratory. The control algorithm is designed with Matlab 7 programming language. The main frequency of the robot driving controller is $230 \mathrm{MHz}$. Personal PC stored as 1GB. According to the above simulation environment, the WIN32 API function CreateFile () function is used to open the PCI device, and the driving control parameters of the continuous software robot are set. The width of the optical sensor output pulse is 2, and the amplitude of the motion control of the software robot is obtained. Within $100 \mathrm{~m}$, the maximum working radius of the driving link is satisfied, and the control performance curve is obtained as shown in figure 3.

Figure 3 shows that with the increase of measurement range, the measurement error of various control methods increases, and the controller designed in this paper can make the error of driving connecting rod to control the motion of soft robot less than $0.408 \mathrm{~m}$. The method has the shortest response time and meets the demand of real-time control.

\section{Conclusions}

In this paper, the optimal control problem of distance measurement for driving link is studied, and a driving control law of continuous soft robot based on dynamic inversion integral error compensation is proposed. The optimal design of robot driving controller is carried out. The algorithm design and the hardware interface design of the controller are described in detail, and the optimization design of the continuous software robot driving controller is realized. The research results show that the controller has better robustness and error correction ability, improves the precision of motion control of software robot, has shorter control response time, and has reliable and stable performance.

\section{References}

[1] Jiang Y, Moseson S, Saxena A. Efficient grasping from RGBD images: Learning using a new rectangle representation[C]//IEEE International Conference on Robotics and Automation. Piscataway, USA: IEEE, 2011:3304-3311.

[2] Krizhevsky A, Sutskever I, Hinton G E. ImageNet classification with deep convolutional neural networks[M]//Advances in Neural Information Processing Systems. Cambridge, USA: MIT Press, 2012:1097-1105.

[3] Maitin-Shepard J, Cusumano-Towner M, Lei J, et al. Cloth grasp point detection based on multiple-view geometric cues with application to robotic towel folding[C]//IEEE International Conference on Robotics and Automation. Piscataway, USA: IEEE, 2010:2308-2315.

[4] LI Ke,MI Jie. Research on Mechanical and Electrical Control Algorithm of Bionic Robot Based on Variable Structure PID [J].Journal of Henan University of Engineering (Natural Science Edition), 2016, 28(2):32-37.

[5] Liao Y H, Li D K, Tang G J. Motion planning of space manipulator system based on a hybrid programming strategy [J]. Journal of Astronautics, 2011, 32(1):98-103.

[6] Darby C L, Hager W W, Rao A V. An hp-adaptive pseudospectral method for solving optimal control problems [J]. Optimal Control Applications \& Methods, 2011, 32(4):476-502.

[7] DU Xuedan, CAI Yinghao, LU Tao, WANG Shuo, YAN Zhe. A Robotic Grasping Method Based on Deep Learning [J]. ROBOT, 2017, 39(6): 820-828,837.

[8] Varley J, Weisz J, Weiss J, et al. Generating multi-fingered robotic grasps via deep learning[C]//IEEE/RSJ International Conference on Intelligent Robots and Systems. Piscataway, USA: IEEE, 2015:4415-4420. 